\title{
LA ORGANIZACIÓN DE LA SOCIEDAD ARGENTINA DE ANTROPOLOGÍA EN SUS PRIMEROS AÑOS
}

\author{
THE ORGANIZATION OF THE ARGENTINE SOCIETY OF ANTHROPOLOGY IN \\ ITS EARLY YEARS
}

\author{
Ana Carolina Arias ${ }^{1}$
}

\begin{abstract}
Este trabajo analiza la organización y los años iniciales de funcionamiento de la primera asociación científica dedicada a las ciencias antropológicas en la Argentina. La Sociedad Argentina de Antropología fue conformada en 1936 por un conjunto heterogéneo de sujetos e intereses. Constituyó un espacio de sociabilidad, de intercambio y de difusión de conocimientos y prácticas vinculadas a las distintas ramas de las ciencias del hombre. También procuró fortalecer estas áreas de estudio como disciplinas científicas. El análisis de la Sociedad permite mostrar el papel que tuvo una organización de origen privado en el fortalecimiento de las ciencias antropológicas en la Argentina en las décadas de 1930 y 1940. Asimismo, muestra cómo las ciencias antropológicas en dichas décadas crecieron gracias a una heterogénea red de relaciones esparcidas en diferentes provincias y territorios nacionales; lo cual fue posible también gracias a la participación activa en la sociedad y en las ciencias antropológicas de aficionados y estudiantes, entre los cuales se observa una amplia presencia de mujeres.
\end{abstract}

Palabras claves: sociabilidad científica, ciencias antropológicas, historia de la antropología, Argentina.

This paper aims to analyze the organization and the early years of the first scientific association devoted to anthropological sciences in Argentina. Formed in 1936 by a heterogeneous group of people with varied interests, the Argentine Society of Anthropology was a space for sociability as well as exchange and dissemination of knowledge and practices linked to different disciplines of human sciences. It also attempted to strengthen the image of these study areas as scientific disciplines. The analysis of the Argentine Society of Anthropology shows the role of a private organization in the strengthening of anthropological sciences in Argentina during the 1930s and 1940s. Furthermore, it shows how the anthropological sciences back then could expand thanks to a heterogeneous network of relationships scattered over different provinces and national territories. This development was also made possible by the active participation in both the society and the anthropological sciences of enthusiasts and students, many of whom were women.

Key words: Scientific networking, anthropological sciences, history of anthropology, Argentina

Hace varias décadas, el historiador Roger Hahn (1971) planteaba un análisis "anatómico" de corporaciones científicas, indagando en las relaciones que hacen al funcionamiento de las mismas y en su articulación con la sociedad en general. Esto implicó un estudio minucioso, algo más que una crónica de los descubrimientos o de la historia social de sus "ilustres" miembros. Así, al utilizar la noción de "anatomía" Hahn apuntó al estudio de las estructuras y las formas, considerando las relaciones entre las diferentes partes del "organismo", digamos, de la institución o sociedad.

Retomando algunas ideas de esta propuesta analítica, este trabajo busca mostrar el papel de las asociaciones privadas en la promoción de la actividad científica. En particular, presenta una "anatomía" de la Sociedad Argentina de Antropología en sus primeros años, prestando atención a su organización y actividades, a sus miembros, a las relaciones con otras sociedades y con los distintos espacios de las ciencias antropológicas. El periodo de análisis se extiende desde la creación de esta asociación en 1936 hasta el año 1945, cuando merma su actividad. Esta fue la primera agrupación de la Argentina dedicada específicamente a las distintas áreas que conformaban la Antropología en aquellos años: Antropología física, Etnología, Etnografía, Lingüística, Arqueología, Antropogeografía y Folklore. El trabajo busca mostrar, entonces, el papel que tuvo una organización de origen privado en el fortalecimiento de las ciencias antropológicas en la Argentina en las décadas de 1930 y 1940.

\footnotetext{
${ }^{1}$ Archivo Histórico de la Facultad de Ciencias Naturales y Museo, Universidad Nacional de La Plata, La Plata, Argentina. anacarolinaarias@yahoo.com.ar
}

Recibido: septiembre 2019. Aceptado: febrero 2020.

http://dx.doi.org/10.4067/S0717-73562021005000201. Publicado en línea: 01-abril-2021. 
Asimismo, a partir de este estudio se da cuenta de la complejidad y de las contradicciones de la mencionada asociación, que pretendía contribuir en la profesionalización de la antropología mediante diversas estrategias. Como se intentará mostrar, las actividades y la continuidad de la Sociedad fueron posibles gracias a que la misma logró reunir -en un ámbito no exento de conflictos- tanto a los "especialistas" como a los "interesados" en las ciencias antropológicas. Para el análisis de estas cuestiones se han relevado una serie de fuentes editas e inéditas. Una parte importante de los fondos consultados proviene de los documentos guardados por la propia Sociedad Argentina de Antropología ${ }^{2}$ y otros son parte de los materiales conservados en el Archivo Fotográfico y Documental del Museo Etnográfico (AFDME) y el Archivo Histórico del Museo de La Plata (AHMLP).

\section{Los Espacios Asociativos de las Ciencias Antropológicas}

La Sociedad Argentina de Antropología fue creada en 1936, siendo la primera asociación especializada en esta disciplina en la Argentina. Hasta esa época, las temáticas antropológicas habían sido tratadas en varias asociaciones científicas del país, donde participaban como socios activos los pocos antropólogos reconocidos. Entre ellas se encontraba la Sociedad Científica Argentina (Babini 1961, Farro 2016), fundada en 1872, y la Junta de Historia y Numismática Americana, iniciada en $1893^{3}$. En las primeras décadas del siglo XX se fundaron otras asociaciones de ciencias naturales, geográficas y americanistas que trataban temas antropológicos en sus reuniones y publicaciones (Pegoraro 2009). Entre ellas se puede mencionar la Sociedad Physis (1911, luego Sociedad Argentina de Ciencias Naturales), integrada por naturalistas, docentes y estudiantes de las carreras universitarias y profesorado de ciencias naturales. Como otras asociaciones, realizaba reuniones periódicas donde se presentaban avances de investigación, publicando la revista Physis desde 1912 (García 2016; García y Podgorny 2000). Esta asociación contempló temas de ciencias antropológicas incluso luego de la creación de la Sociedad Argentina de Antropología. En sus reuniones y en la revista se expusieron y publicaron numerosos artículos de antropología, los cuales dejarán de tener presencia hacia 1942. Hasta ese año, la Revista Physis incluyó entre sus secciones la sección de Antropología, Etnografía y Arqueología.

Otra asociación importante de promoción de algunas áreas de las ciencias antropológicas fue la Sociedad Geográfica Argentina "GAEA", fundada en 1922 con el propósito de promover las ciencias geográficas en sus variadas disciplinas: etnografía, geodesia, topografía, cartografía, biogeografía, geología, geofísica, climatología y oceanografía, entre otras (Curto et al. 2008; García 2016). Fue la primera asociación científica argentina presidida por una mujer, Elina González Acha de Correa Morales (1861-1942) ${ }^{4}$. En 1922, la sociedad comenzó a editar los Anales de GAEA, Sociedad Argentina de Estudios Geográficos, donde predominaron los trabajos geológicos, junto con otros temas relacionados con la geografía física, la cartografía, la toponimia y la antropogeografía. Desde 1934 editó un Boletín de GAEA, Sociedad Argentina de Estudios Geográficos y otras publicaciones (Curto et al. 2008, García 2016). Esta asociación tuvo estrechas vinculaciones con las ciencias antropológicas y con la Sociedad Argentina de Antropología, puesto que ambas compartían una gran cantidad de miembros y un conjunto de intereses científicos (Arias 2019). En relación a ello, Lascano y Curto (2013) hablan de una "expansión de la antropología" hacia la Sociedad Argentina de Estudios Geográficos, que recibió subsidios para financiar viajes de estudio que abarcaban temas geográficos y arqueológico-etnográficos. La sociedad también participó en la "Semana de la antropología" y dedicó algunas sesiones de la "Semana de Geografía" a las investigaciones antropológicas.

Estas organizaciones mantuvieron vínculos con la Sociedad Argentina de Antropología, compartiendo miembros y temáticas antes y después de la creación de la misma. De hecho, quienes fundaron la Sociedad Argentina de Antropología poseían trayectorias de participación en diversas sociedades, tanto nacionales como internacionales, lo cual permitió capitalizar esa experiencia en propuestas y acciones concretas, como las sesiones de comunicaciones, la semana de la Antropología y las publicaciones.

En cuanto a las asociaciones dedicadas específicamente a las ciencias antropológicas, la creación de las mismas a nivel internacional se había promovido desde la segunda mitad del siglo XIX. Al fundarse la Sociedad Argentina de Antropología, se sumó al conjunto de 68 asociaciones análogas existentes en el resto del mundo (Podgorny 2004). En Latinoamérica, se pueden mencionar algunas iniciativas en el siglo XIX pero la mayoría suceden en las primeras décadas del XX: en Chile, en 1878 se fundó la Sociedad Arqueológica de Santiago, la cual sobrevivió unos pocos años. Luego, en 1909, se estableció la Sociedad del Folklore Chileno y más adelante se crearon la Sociedad de Arqueología e Historia "Francisco Fonck" de Viña del Mar (1937) y la Sociedad Arqueológica de La Serena (1944) (Gänger 2014, Mora Nawrath 2016). Uruguay contaba con una asociación de aficionados desde 1927, la Sociedad de Amigos de la Arqueología, creada con el fin de "agrupar en torno suyo todos los elementos que en el país se sintieran inclinados al culto de las cosas arcaicas" arqueología como la paleontología. En 1930 se creó la Sociedad Arqueológica de Bolivia, por iniciativa del ingeniero militar austro-húngaro Arthur Posnansky 
(1874-1946) junto con 11 selectos socios (Browman 2007). En 1937 se creó la Sociedad Mexicana de Antropología, conformada por antropólogos, lingüistas, etnólogos, arqueólogos e historiadores ${ }^{6}$. En 1941, en la Facultad Nacional de Filosofía de la Universidad del Brasil, tuvo lugar la creación de la Sociedad Brasilera de Antropología y Etnología?.

\section{La Sociedad Argentina de Antropología}

La Sociedad Argentina de Antropología fue la primera asociación especializada en las ciencias del hombre en el país. Su creación sucedió en un contexto de expansión institucional y de renovación de cargos en las ciencias antropológicas. En los inicios de la década de 1930 se produjo un recambio de quienes ocupaban los cargos docentes y científicos de las instituciones de Buenos Aires y La Plata, tras la jubilación y/o la muerte de las figuras que ocuparon esas posiciones desde principios del siglo sin formar discípulos ni consolidar grupos de trabajo (García 2010; Podgorny 2004). Paralelamente, se fomentaron las instituciones académicas y los museos en las provincias argentinas (García 2011), y la promoción del estudio de las culturas regionales, incentivando el estudio de nuevas localidades y temáticas, junto con el estímulo a estas disciplinas a través de cursos y de la formación docente.

En 1936 se fundó la Sociedad Argentina de Antropología "con el objeto de coordinar la labor de los especialistas en las ciencias del hombre, realizar investigaciones en el terreno de acuerdo con planes orgánicos y normas precisas, y promover, asimismo, una intensa penetración cultural tendiente a difundir y divulgar los resultados científicos obtenidos".

Sus miembros, al elaborar el acta de fundación, definieron que la Antropología abarcaba "cualquiera de las disciplinas que constituyen las ciencias del hombre (Antropología física, Etnología, Etnografía, Linguiística, Arqueología, etc.)", siendo "especialistas" aquellas personas dedicadas a "la investigación en alguna de las ramas de la Antropología, como objeto principal de su actividad, y que además de haber producido obras meritorias, han seguido estas actividades con carácter profesional". Es decir, para entonces, se reconocía que en el país había un pequeño grupo de personas dedicadas a la investigación antropológica de forma profesional y que aspiraba a generar un espacio propio de sociabilidad científica.

Esta asociación se constituyó con siete socios en una reunión en el Museo Mitre de Buenos Aires. Sus miembros fundadores ocupaban las cátedras de ciencias antropológicas de las universidades de Buenos Aires y de La Plata, y del Instituto Nacional del Profesorado, y también los puestos de jefes de división en los museos de Buenos Aires y La Plata. Sus nombres son conocidos en la historia de la antropología argentina: Francisco de
Aparicio $^{10}$, Eduardo Casanova ${ }^{11}$, José Imbelloni ${ }^{12}$, Fernando Márquez Miranda ${ }^{13}$, Enrique Palavecino ${ }^{14}$, Félix Outes $^{15}$ y Milcíades Vignati ${ }^{16}$. Como primer presidente fue elegido Outes, quien dirigía en ese momento el Museo Etnográfico; como secretario Casanova, Jefe de la Sección de Arqueología del Museo Nacional de Historia Natural; en tanto que la tesorería fue cubierta por Vignati; y como director de publicaciones se nombró a Imbelloni, profesor titular de Antropología y Etnografía de la Universidad de Buenos Aires (Arias 2019; Podestá 2007). Después de la primera reunión se agregaron tres socios: Antonio Serrano, Duncan L. Wagner y Emilio R. Wagner ${ }^{17}$.

Como en otras asociaciones, la organización de esta Sociedad se fue ajustando año a año, de acuerdo a la cantidad y participación de sus miembros, y a las propuestas que surgieron en su seno. Respecto de las formas de membresía, en el primer estatuto (1936) se establecieron dos categorías de socios: activo y adherente. La primera se reservó para los llamados "especialistas", es decir, aquellos considerados como "profesionales", dedicados a la enseñanza e investigación como empleados de algún museo o universidad. Con el abono de la cuota correspondiente, los socios activos recibían sin cargo las publicaciones de la Sociedad (la revista Relaciones y el Boletín) y podían concurrir a todos los actos que la misma organizara. La segunda categoría de asociado, pagaba una cuota más reducida y era para "todas las personas que se interesen por las actividades de la Sociedad"18.

En mayo de 1937 se publica el primer balance anual de la asociación y se propone un nuevo estatuto, en el cual se agregan cuatro nuevas categorías de socios: "estudiantes", "honorarios", "correspondientes" y "protectores". Los socios estudiantes podían ser los alumnos de los institutos superiores en los que se impartía alguna enseñanza relacionada con las ciencias antropológicas, no pagaban cuota pero igual recibían el Boletín, accedían a un precio especial de la revista Relaciones y podían asistir a los actos organizados por la asociación. Podían ser socios honorarios personas o instituciones con "destacada" actuación en los estudios antropológicos; socios correspondientes personas o instituciones residentes en el extranjero que se dedicaran a la investigación antropológica; y socios protectores personas o instituciones que contribuyeran al sostenimiento de la Sociedad con una cuota mínima de 100 pesos anuales, o que hicieran una donación superior a 1.000 pesos $^{19}$. Asimismo, se redefinió la condición de socio activo, extendiéndose a cualquier persona que se "interese" por las actividades de la Sociedad. Con ello, se amplió la base social de los miembros activos, lo cual además se dio en paralelo a la reducción del importe de la membresía ${ }^{20}$. Este cambio se debió a las limitaciones para asociarse de modo "activo" en el reglamento inicial, generando un "serio inconveniente para el progreso de 
la Sociedad"21. El estatuto de 1937 permitió incrementar la cantidad de afiliados y con ello obtener mayores ingresos de dinero a pesar de la reducción de la cuota anual. Además, la Comisión Directiva de la Sociedad consideró que dicho incremento permitía mostrar públicamente una mayor consolidación de la asociación y solicitar subsidios a distintos organismos. Así, tras un año de funcionamiento, a los pocos "especialistas" que fundaron la Sociedad se sumaron los "interesados" en las ciencias antropológicas, incluidas varias mujeres que participaron activamente en la misma ${ }^{22}$. Por otra parte, los socios muestran cierta dispersión territorial en los primeros años: si bien el 70\% de ellos vivían en Buenos Aires, se registran miembros en las ciudades de La Plata, Tucumán, Santiago del Estero, Santa Rosa, Godoy Cruz, Mendoza, Santa Fe, Rosario, Concepción (Provincia de San Juan), San Juan, Salta y Chaco 23 .

Como se observa en las figuras, la membresía fue creciendo a partir de 1937. Los datos provienen de las comunicaciones publicadas por la Sociedad y permiten observar dos momentos donde se incorporan gran cantidad de miembros (en todas las categorías), uno en 1936-193724 y otro en 1941-1942 $2^{25}$. El primer momento se corresponde con el cambio de estatuto y con la ampliación de las categorías de socios. El segundo podría vincularse con un auge de la asociación, en la cual se han consolidado diferentes actividades y se ha promovido activamente el ingreso de nuevos miembros; y en relación también a la continua promoción de las ciencias antropológicas. Los períodos con mayor incorporación de socios activos fueron 1937-38 y 1941-42 (Figura 1), mientras que la mayor parte de socios estudiantes se da en el periodo 1941-42 (Figura 2). En conjunto, las nuevas incorporaciones de socios y socias muestran un aumento en 6 años que triplicó la nómina inicial (Figura 3). Por supuesto, también en estos años hubo personas que abandonaron la membresía o que fueron dadas de baja por falta de pago de las cuotas societarias $^{26}$. Esto último era poco frecuente, puesto que constantemente se ofrecían plazos y opciones de actualización de los pagos pendientes ${ }^{27}$.

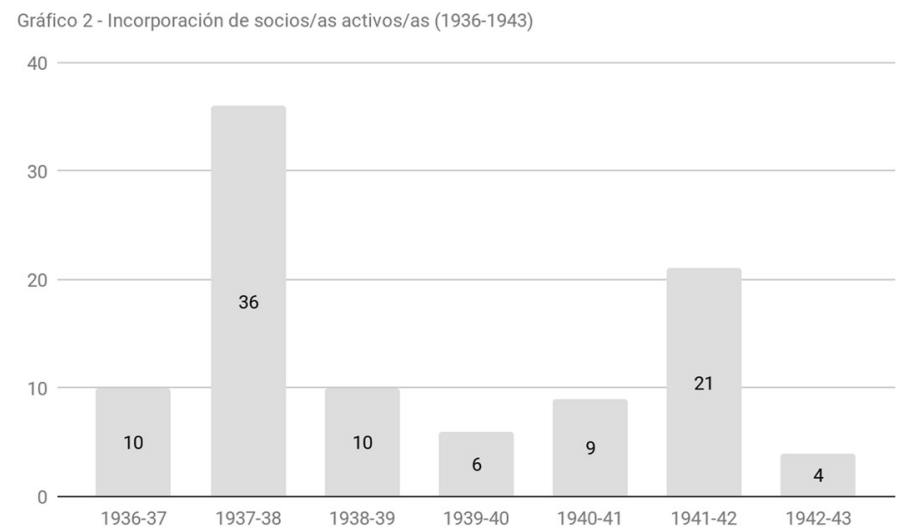

Figura 1. Membresía acumulada (1936-1943) .

Total membership (1936-1943).

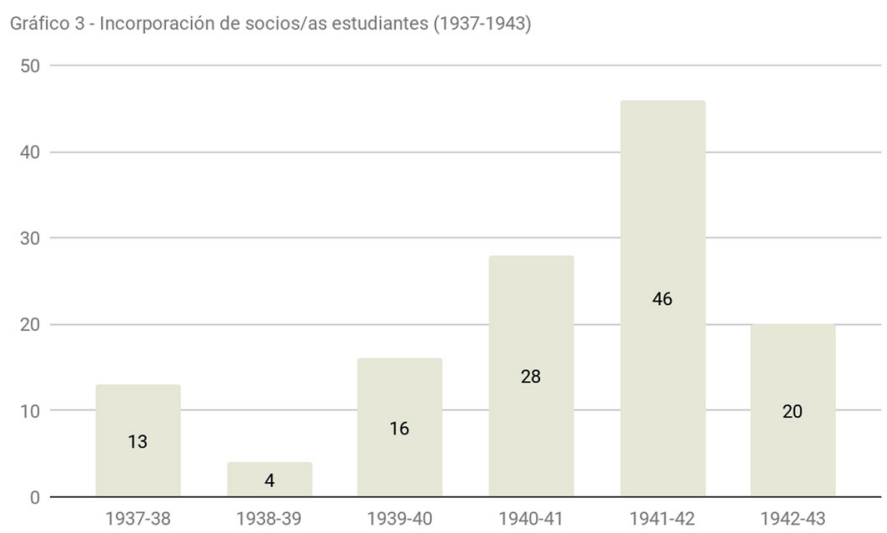

Figura 2. Incorporación de socios/as (1936-1943).

Incorporation of members (1936-1943). 


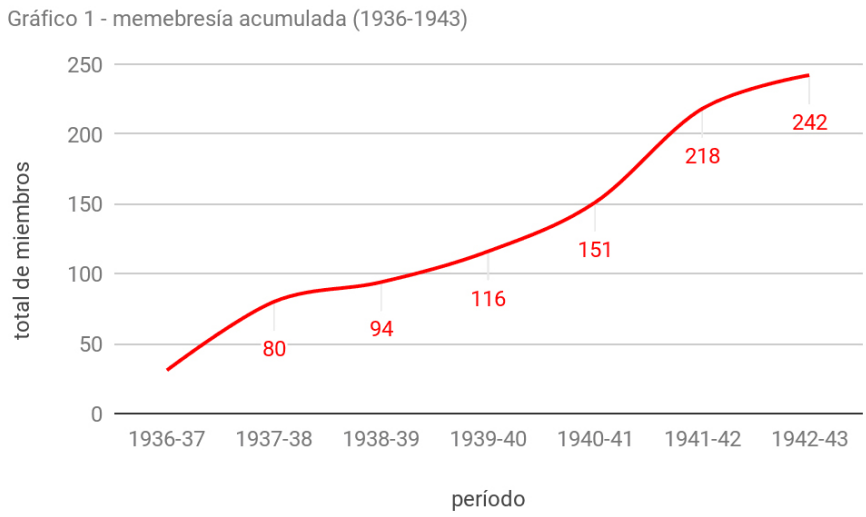

Figura 3. Incorporación de socios/as estudiantes (1937-1943).

Incorporation of student members (1937-1943).

El aumento de los afiliados también garantizó el funcionamiento de los espacios de comunicación, permitiendo contar con expositores y público para las actividades. En particular, las exposiciones orales de trabajos contaron con un auditorio compuesto mayormente por estudiantes, según se señala en los registros de eventos en el Boletín o en las Memorias Anuales de la Sociedad. Por ejemplo, en una disertación en 1943 sobre "relevamiento de pinturas y grabados aborígenes", se aclara que es "de carácter didáctico dirigida especialmente a los alumnos que generalmente asisten a estas conferencias" ${ }^{\prime 28}$. De hecho, como se puede observar en la Figura 4, la cantidad de estudiantes se fue incrementando, con una mayoría creciente de mujeres, las que constituían más del $50 \%$ de la membresía estudiantil a partir del periodo $1938-39^{29}$.

Gráfico 4. Incorporación de socios/as activos y estudiantes, 1936-1943

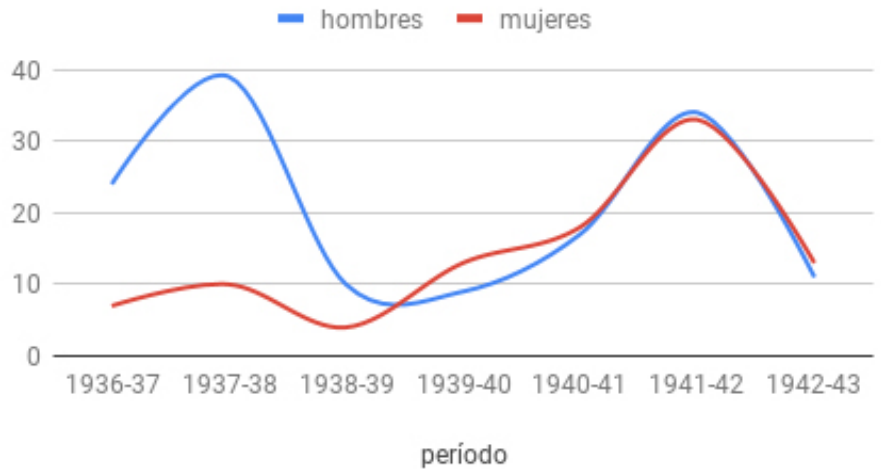

Figura 4. Incorporación de socios/as activo/as y estudiantes (1937-1943).

Incorporation of active members and students (1937-1943). 


\section{Las actividades de la Asociación}

La Sociedad desarrolló distintas actividades, como publicaciones, reuniones de comunicación de trabajos y la llamada "Semana de la Antropología", con el fin de la "intensificación de los estudios antropológicos". Como otras asociaciones científicas, las "sesiones científicas" de presentación y discusión de trabajos constituyeron una de las actividades centrales, puesto que "llevan de inmediato a la práctica uno de los propósitos más elevados de penetración cultural”"30 de la sociedad. Estas reuniones se realizaron desde 1937 con una frecuencia generalmente mensual entre abril y diciembre ${ }^{31}$. En algunas ocasiones se distanciaron más de un mes al intercalarse con la Semana de la Antropología. En las disertaciones se presentaban resultados o avances de trabajos científicos, usualmente acompañadas con proyecciones o algunos objetos a modo de ejemplo. De las mismas participaban los socios activos y en ocasiones los estudiantes. A estas reuniones podían concurrir los asociados, sus familiares y personas invitadas especialmente por el Consejo Directivo.

Otra de las propuestas impulsadas desde el inicio fue la realización de jornadas científicas: en mayo de 1936 Palavecino propuso la realización de unas "Jornadas Antropológicas" y la Comisión Directiva le encargó que realizara "los sondeos previos y la preparación de un anteproyecto de estructuración de las mismas" 32 . Unos meses después contaba con una veintena de propuestas de trabajos y se creó una comisión para la organización y propaganda de las jornadas, conformada por Palavecino, Vignati y Aparicio; pero las mismas no se llevaron a cabo. En septiembre de 1937 Aparicio retomó esa idea y la planteó con el nombre de "Semana de la Antropología" 33 , la cual consistiría en exposiciones orales de trabajos de "especialistas", quienes debían ser autorizados por la comisión científica de la Sociedad ${ }^{34}$. Los trabajos eran presentados y luego discutidos con el público presente. En general, estos trabajos eran luego enviados para su publicación en la revista de la sociedad. La "Semana" se proyectó de modo anual, aunque no todos los años logró concretarse. La primera se llevó a cabo en el Museo Etnográfico de Buenos Aires, entre el 29 de noviembre y el 4 de diciembre de 1937, y las disertaciones fueron sobre diferentes temáticas. La segunda se realizó dos años después, también en Buenos Aires y trató un único tema: "Los Primitivos Habitantes de Santiago del Estero". La tercera semana se realizó en Mendoza en 1941, con el fin de "estimular la labor que en el campo de las ciencias antropológicas" desarrollan ciertas universidades ${ }^{35}$. La cuarta semana se realizó nuevamente en Buenos Aires, en 1944, y tuvo como tema de discusión "La antigua provincia de los Diaguitas" ${ }^{36}$. La quinta semana ocurrió en La Plata (1945) y las siguientes fueron en Buenos Aires, con excepción de la sexta realizada en Santa Fé (1951) y la séptima y novena que realizaron sesiones en Buenos Aires y en La Plata. En total se realizaron 10 "Semanas" entre 1936 y $1956^{37}$.

Otra de las actividades de la Sociedad fueron las publicaciones. Como señala Lopes (1999), el emprendimiento editorial constituyó una labor esencial en las actividades de las instituciones y asociaciones científicas, dándoles visibilidad pública más allá de los espacios locales donde actuaban. La edición, distribución y canje de publicaciones permitía tanto a las instituciones como a sus investigadores el ingreso a los circuitos internacionales de canje de bibliografía y a distintas redes de intercambio en el ámbito local e internacional (García 2010). Los resultados de trabajos de campo y de laboratorio, así como las colecciones y catálogos, cobraban mayor valor si eran publicados y puestos en circulación, permitiendo compartir y circular universalmente la producción de conocimientos (Pyenson y Sheets-Pyenson 1999, Podgorny 2002). La Sociedad Argentina de Antropología organizó dos publicaciones: la revista Relaciones, destinada a publicar trabajos de investigación, y el Boletín que se dedicaba a publicar noticias vinculadas a la sociedad, resúmenes de comunicaciones, entre otros.

Relaciones tuvo su primer número en 1937, los siguientes serán en 1940, 1942 y $1944^{38}$. La misma contaba con una tirada habitual de 500 ejemplares y se distribuía de forma gratuita a los socios activos. La venta al público general tenía un valor de 20 pesos en 1938 y de cinco pesos para los socios estudiantes. En 1943 el valor de los tomos era de 15 pesos. Los temas de esta publicación incluyeron informes sobre excavaciones y objetos arqueológicos de diferentes regiones del país y de países vecinos, estudios sobre documentos históricos y etnohistóricos, hallazgos de cráneos y esqueletos, trabajos sobre grupos sanguíneos y taxonomía humana. El espacio de la publicación contribuía con la definición del universo de saberes y prácticas socialmente legítimos que contribuyeron a producir y reproducir programas de pensamiento; y con la creación de un público inmediato, formado por estudiantes y futuros docentes, que posibilitaron la reproducción ampliada de la práctica científica y su divulgación (García 2010; Lopes 1999).

Por su parte, el Boletín era una publicación cuyo objetivo consistía en "difundir los diversos aspectos de la obra cultural realizada por la Sociedad". En el mismo se publicaban resúmenes de las reuniones de comunicaciones y de los trabajos presentados en la Semana de la Antropología, avisos a los socios, noticias de varias instituciones, hallazgos científicos y otras novedades en torno a las ciencias antropológicas y a las actividades de la asociación. Si bien el Boletín estuvo proyectado desde inicios de la Sociedad, se publicó por 
primera vez en 1942, continuando en sus primeros años de forma regular hasta 1945 y con un único ejemplar en el año 1963.

Para concretar las publicaciones, la Sociedad solicitó diversos subsidios a diferentes reparticiones públicas. En 1936, se dirigió a la Comisión Nacional de Cultura, indicando problemas económicos por las pocas cuotas recaudadas "dado el limitado número de sus socios y adherentes, [la Sociedad] tropieza con dificultades insalvables para iniciar sus publicaciones que han de exteriorizar su obra, ha creído que tal vez la comisión que Ud. tan dignamente preside podría prestar su valioso apoyo a nuestra acción cultural" ${ }^{\prime 39}$. Una carta similar fue enviada al Presidente de la Comisión de Presupuesto de la Cámara de Diputados de la Nación, esta vez solicitando una suma específica de tres mil pesos. Finalmente, a fines de 1937 se logra un subsidio anual mediante una asignación en el presupuesto correspondiente al Ministerio de Relaciones Exteriores y Culto, el cual se mantuvo hasta $1944^{40}$. Luego se gestionaron publicidades y otros pedidos de ayuda económica para el accionar de la Sociedad, especialmente para financiar las publicaciones ${ }^{41}$.

Otras actividades desarrolladas por esta asociación incluyeron excursiones a localidades que "pudieran ser de interés desde el punto de vista antropológico" ${ }^{2}$, cenas de camaradería y clases prácticas para los socios, como por ejemplo de fotografía científica ${ }^{43}$. También se ofrecieron cursos breves para profesores de enseñanza secundaria, a través del Ministerio de Justicia e Instrucción Pública, motivados por las "frecuentes comunicaciones solicitando bibliografía e informaciones acerca de las culturas de los aborígenes de América" y con el fin de "colaborar al mejor conocimiento de las culturas de los aborígenes de nuestro territorio" ${ }^{44}$.

Por otra parte, en el año 1940 se solicitó a la Comisión Nacional de Cultura que incluya a la Antropología entre las disciplinas que premiaba. También se le pidió que modifique la forma en que se conformaban los jurados para los premios regionales: en vez de seleccionar los jurados por su vinculación con la región, quienes según la Asociación no siempre estaban capacitados para juzgar las obras cuando las mismas eran "de carácter histórico", se insistía que debían ser seleccionados y agrupados por temáticas o por especialidades ${ }^{45}$. La sugerencia fue tomada por la Comisión de Cultura para el nombramiento de jurados del año siguiente ${ }^{46}$.

Como parte de las actividades, la Asociación organizó comisiones para realizar trabajos específicos, tales como "precisar y fijar el vocabulario técnico de antropología y ciencias afines", o la realización de comentarios bibliográficos para que "la Sociedad hiciera conocer la bibliografía que, antigua o moderna, fuera poco conocida" ${ }^{\star 47}$. En relación con ello, la Sociedad se presentaba como un espacio para "vigorizar el espíritu de solidaridad entre los que se dedican a estos estudios, y coordinar la investigación, haciéndola más proficua mediante la acción conjunta de los antropólogos y de todos aquellos que se sienten atraídos por los problemas que presentan las Ciencias del Hombre." ${ }^{38}$ Sin embargo, en su seno se dieron también disputas y discusiones, manifestadas en ocasiones como desacuerdos sobre la interpretación de distintas investigaciones.

\section{Debates y discusiones: delimitar el campo de las ciencias antropológicas}

Las discusiones sobre temas de gran interés muestran la forma en que la Sociedad Argentina de Antropología buscó participar en la delimitación del campo de las ciencias antropológicas. Sus actividades, como vimos, apuntaban a generar espacios de diálogo y consenso sobre diferentes aspectos. Sin embargo, también hubo disputas y conflictos orientados a generar diferenciaciones entre las "opiniones especialistas" y las que -supuestamente- no lo eran.

$\mathrm{Al}$ respecto, destacan dos cuestiones ampliamente debatidas. Por un lado, los hallazgos realizados por los hermanos Wagner en Santiago del Estero. Los mismos alcanzaron amplia difusión a través de la publicación de su libro "La civilización Chaco-Santiagueña y sus correlaciones con las del Viejo y Nuevo Mundo" $(1934)^{49}$ y, la Sociedad organizó la segunda "Semana de la Antropología" en torno a estas discusiones. Los trabajos de la misma se publicaron luego en el segundo tomo de Relaciones (1940). Específicamente, se discutió sobre la interpretación de los hallazgos arqueológicos realizados por los hermanos Wagner. Frente a un tema en el cual habían intervenido "hombres de letras, estudiosos de disciplinas heterogéneas, periodistas, políticos y turistas", la Sociedad Argentina de Antropología daba a conocer "la opinión de los especialistas argentinos" (Aparicio 1940:2). Como resultado, los diferentes participantes de esta discusión, rechazaban las hipótesis planteadas por los Wagner y -según Martínez, Taboada y Auat (2011)- con ello los "expulsaban" del campo académico.

Por otro lado, la cuestión de los hallazgos realizados en las zonas aledañas a la ciudad de Santa Fe, especialmente en el paraje llamado Arroyo Leyes, de inicios de la década de 1930, que generaron un amplio interés. Las piezas cerámicas encontradas suscitaron debates en torno a su clasificación y autenticidad (Bonin 2008; Ceruti 2012, Podgorny 2014). En la discusión intervinieron diferentes arqueólogos, coleccionistas y aficionados, entre ellos, Francisco de Aparicio, Antonio Serrano, Félix Outes, los sacerdotes jesuitas Raúl Carabajal y Guillermo Furlong, Amelia Larguía, Manuel Bousquet, Fernando Mántaras, entre otros 
(Arias 2019). Varios de ellos eran miembros de la Sociedad Argentina de Antropología, la que en la década de 1940 proyectó una expedición a la región en conjunto con el Departamento de Estudios Etnográficos y Coloniales de Santa Fe, creado en 1940, a cargo de Agustín Zapata Gollán (1895-1986). Si bien la Asociación consiguió que la provincia de Santa Fe le otorgara un subsidio de tres mil pesos para realizar las excavaciones, por diversos desacuerdos para ejecutar la expedición, la misma se fue postergando y finalmente no se realizó ${ }^{50}$.

Así, la Sociedad Argentina de Antropología se posicionó como una voz autorizada para debatir y resolver ciertas problemáticas científicas, buscando constituirse como la referencia de un campo disciplinar en conformación; con prácticas aún heterogéneas y débilmente reguladas. Precisamente, una de las cuestiones que se discuten en estos años es la práctica ocasional de la arqueología, con fines comerciales o como una "distracción turística", la cual resulta perjudicial para las investigaciones sistemáticas:

\begin{abstract}
"la mayoría de los yacimientos arqueológicos del noroeste argentino adolecen de la falla común de haber sido vandalizados por manos ignaras de buscadores de tesoros, de arqueólogos ocasionales y de turistas. Estas tres plagas obran de consuno, para lograr un efecto final: la inutilización del yacimiento para toda investigación sistemática y exhaustiva" ${ }^{\circ 1}$.
\end{abstract}

Como se observa, la Sociedad buscó posicionarse en un papel central en la distinción entre especialistas y no especialistas (no siempre con éxito), así como en la promoción de investigaciones acordes a sus propios parámetros de sistematicidad y profesionalismo. Al mismo tiempo, el propio funcionamiento y continuidad de la asociación estuvo íntimamente ligado a la heterogeneidad de sus miembros.

\section{Conflictos internos y externos}

Los acontecimientos políticos de mediados de la década de 1940 no fueron ajenos a las preocupaciones de la Sociedad Argentina de Antropología, en cuyo seno se dieron discusiones respecto de los cambios políticos ocurridos a nivel estatal y su posible incidencia sobre el funcionamiento de la asociación. Como han señalado diversos autores, en 1943 se inició un proceso que comenzó con el golpe militar de junio y que tuvo impacto algunos meses después en el ámbito académico porteño, cuando se decretó la intervención de la universidad de Buenos Aires (Buchbinder 1997, 2005; Halperín Donghi 1962). En la Universidad de Buenos Aires, y especialmente en la Facultad de Filosofía y Letras, un porcentaje significativo de profesores fue cesanteado $u$ obligado a renunciar, se modificaron las estructuras de investigación y los planes de estudio se pusieron en revisión o reforma. A comienzos de 1945, los conflictos entre grupos reformistas y católicos se agudizaron y si bien las autoridades universitarias intentaron limitar las polémicas de carácter político en el interior de las instituciones, "no hubo forma de sustraerse al conflictivo proceso político que se inició entonces" (Buchbinder 1997:157).

En relación con esta situación, en la sesión del Consejo Directivo del 12 de septiembre de 1945 se decidió postergar la realización de la Semana de la Antropología y el entonces Presidente de la asociación, Francisco de Aparicio, propuso el siguiente comunicado para dar a publicidad:

"La Comisión Directiva de la Sociedad Argentina de Antropología, en vista de las manifestaciones repetidas de sus miembros más conspicuos que aclaran no poder participar en certámenes científicos porque la intranquilidad reinante en las horas angustiosas que vivimos les niega la calma indispensable para toda labor de estudio e investigación, y teniendo en cuenta que este estado de ánimo se manifiesta asimismo en el público que concurre habitualmente a reuniones científicas, el cual ha faltado casi en absoluto en diversos actos celebrados últimamente en sociedades afines, resuelve suspender la Semana de la Antropología que debía celebrarse en el próximo mes de octubre y todas sus actividades públicas durante el corriente año" 52 .

Esta declaraciónfue objetadaporel "carácterpolítico" de la misma. Casanova propuso modificaciones a la declaración, e Imbelloni manifestó que "la sociedad era de carácter científico y que nada tenía que ver con hechos políticos", declaración apoyada por Romualdo Ardissone ${ }^{53}$. Por último, la secretaria María de las Mercedes Constanzó ${ }^{54}$ señaló que no sería necesario dar noticia de la suspensión del evento puesto que aún no se había anunciado la realización del mismo. Estas diferencias personales, junto al clima político y de incertidumbre de la época, contribuyeron con la disminución de actividades de la Sociedad Argentina de Antropología.

A comienzos de 1946 se realizó una nueva intervención sobre las universidades decretada por el Poder Ejecutivo Nacional (Buchbinder 1997). Entre 1946 y 1947 la oposición reformista fue confrontada en todas las universidades nacionales por medio de la cesantía de 423 docentes, al tiempo que se generaron las condiciones para que otros 823 renunciaran voluntariamente o en solidaridad con los primeros, oponiéndose a la limitación de funciones en las tareas de docencia e investigación y/o como crítica al recorte de la autonomía. Los debates frente a las intervenciones tuvieron expresión parcial en las diferentes instituciones y espacios académicos (Buchbinder 1997; Soprano 2009). En el caso de 
la Sociedad Argentina de Antropología, a este contexto académico y político problemático se le sumaron dificultades económicas, especialmente la escasez de recursos para afrontar las publicaciones.

En abril de 1947, el decano interventor de la Facultad de Filosofía y Letras de Buenos Aires dispuso que las reuniones de la Sociedad Argentina de Antropología en el Museo Etnográfico debían comunicarse con cinco días de anticipación, expresando también los temas que serían tratados en las mismas. Asimismo, toda noticia enviada a los periódicos desde la asociación debía realizarse por vía de la Secretaría de la Facultad. Estas disposiciones fueron entendidas como "tutoría" y "censura" por los miembros del Consejo Directivo de la Sociedad y se propuso el traslado de su sede a la Sociedad Argentina de Estudios Geográficos GAEA ${ }^{55}$. Las siguientes reuniones del Consejo Directivo se hicieron allí, aunque se fueron espaciando en el tiempo, disminuyendo la frecuencia a dos o tres reuniones por año en lugar de los habituales encuentros mensuales. En el Boletín Bibliográfico de Antropología Americana publicado en 1947 se señala la "escasez" de actividades y la disminución de la vitalidad de la Sociedad Argentina de Antropología $a^{56}$. Asimismo, durante este año no se editó ninguna publicación, aunque se celebraron algunas reuniones de comunicaciones. Estos encuentros serán en 1948 la única actividad que la Sociedad estuvo en condiciones de organizar, invitándose "aún a aquellos que no tengan antecedentes" y a "personas ajenas a la Sociedad" ${ }^{7}$. Las reuniones del Consejo Directivo, además de distanciarse cada vez más en el tiempo, tendrán cada vez menos participantes. En diciembre de 1948 se reunieron cinco personas que decidieron convocar una asamblea extraordinaria para la renovación de las autoridades, la cual ocurrió en julio de 1949, con poca presencia de socios ${ }^{58}$. En 1950 se retoman algunas actividades, con la intención de volver a publicar el Boletín, lo cual no ocurrió hasta 1963. Si bien algunas actividades se continuaron hasta 1956, como las Semanas de la Antropología, la participación fue escasa y ciertos "problemas de índole interna" ${ }^{59}$ dificultaron la vitalidad de la asociación.

De forma paralela al decaimiento de actividades de la Sociedad surgen otras asociaciones donde se fomentan las ciencias antropológicas. En 1943 se creó un "círculo de alumnos" ${ }^{\circ 0}$ en la Facultad de Filosofía y Letras de Buenos Aires llamado AKIDA ${ }^{61}$, el cual proponía una labor colectiva y contribuyó a recaudar fondos para viajes de entrenamiento organizados en conjunto con los profesores de la Facultad. Por otra parte, en 1946 se creó la Sociedad Argentina de Americanistas, la cual se definió como una "entidad de carácter exclusivamente cultural, cuyos socios realizan una labor desinteresada, sin otra finalidad que el adelanto de la Americanística en general y de las ciencias particulares predilectas por cada uno de ellos" ${ }^{\prime \prime 2}$. Esta asociación realizaba sus sesiones plenarias y sus conferencias en el Instituto de Arte Americano de la Facultad de Arquitectura de la Universidad de Buenos Aires. Muchos de los miembros fundadores de la Sociedad Argentina de Antropología participaban de estas y de otras sociedades.

\section{Palabras Finales}

En este trabajo se intentó dar cuenta de la "anatomía" de la Sociedad Argentina de Antropología en sus primeros años, poniendo el foco en su organización y en sus actividades, especialmente en sus primeros años de funcionamiento. El análisis de la Sociedad permitió mostrar el papel que tuvo una organización de origen privado en el fortalecimiento de las ciencias antropológicas en la Argentina en las décadas de 1930 y 1940.

Como se ha dicho, la Sociedad Argentina de Antropología fue creada en 1936 por un grupo reducido de "especialistas" en las ciencias del hombre. Esta asociación proveyó a sus miembros de un espacio en común para la comunicación de resultados de las investigaciones científicas, tanto a través de intercambios particulares como a través de publicaciones, boletines, conferencias y exposiciones. Asimismo, contribuyó con el soporte material de diferentes investigaciones, organizando expediciones y obteniendo subsidios estatales para tales fines. El establecimiento de encuentros y reuniones periódicas, el calendario de actividades generado a partir de las "sesiones de comunicaciones" y las "Semanas de la antropología", permitía a los socios un espacio donde dar a conocer y discutir sus trabajos, posibilitando también la creación de alianzas, así como la expresión de críticas y controversias.

Asimismo, la Sociedad Argentina de Antropología se posicionó como una voz autorizada para intervenir en ciertas discusiones y en sus primeros años logró conformarse como un espacio de referencia en las diferentes disciplinas que en ese momento se consideraban parte de las Ciencias Antropológicas: Antropología física, Etnología, Etnografía, Linguiística, Arqueología, Antropogeografía, Folklore, entre otras. Esta autoridad también permitió a la Sociedad encargarse del dictado de cursos específicos para maestros y la intervención en las designaciones de premios nacionales, entre otros aspectos.

A diferencia de lo que una parte de las historias disciplinares muestran, al analizar las actividades en los primeros años de esta Sociedad se observa que las ciencias antropológicas en estos años crecieron gracias a una heterogénea red de relaciones esparcidas en diferentes provincias y territorios nacionales (y no solo en el circuito porteño-rioplatense). Ello fue posible también gracias a la participación activa en la sociedad y en las ciencias antropológicas de aficionados, interesados 
y estudiantes, entre los cuales se observa una amplia presencia de mujeres.

Durante su primer decenio, la asociación estuvo conformada por diversos sujetos, intereses y saberes: la historia, la geografía, la arqueología, el folklore, la etnografía, la etnobotánica, la enseñanza en diferentes niveles, entre otros. La distinción creada por los fundadores de la Sociedad entre "especialistas" e "interesados" resultó poco provechosa para la incorporación de nuevos miembros, y tuvo que ser rápidamente modificada para poder incrementar la presencia de socios activos en sus primeros años. Ello permite relativizar las distinciones entre "aficionados/ profesionales", sin considerarlas como categorías generales implícitas desde el comienzo de una investigación, sino como el producto de conflictos, negociaciones y compromisos entre categorías de actores en un contexto determinado (Guillemain y Richard 2016).

Como señala Podgorny (2005), el estudio de los modos particulares de sociabilidad científica implica también atender a su relación con cierta definición institucional y su contexto material y social, teniendo en cuenta que tanto las ideas como las instituciones son producto de las acciones, elecciones y estrategias de actores concretos en el marco de contextos específicos. En este sentido, la Sociedad Argentina de Antropología constituyó un espacio de sociabilidad, de intercambio y difusión de conocimientos y prácticas; que desarrolló y promovió estrategias para el fortalecimiento de las ciencias antropológicas como disciplinas científicas. Asimismo, se articuló con diversas asociaciones e instituciones, especialmente las universidades, generando relaciones entre los espacios de cátedra, de sociabilidad y camaradería y de investigación, tanto en los museos como en los viajes y expediciones de campo.

Agradecimientos: A CONICET por una beca postdoctoral, a Susana García por las lecturas de diferentes versiones de este manuscrito, a la Universidad de La Plata por el financiamiento para realizar esta investigación y a las personas que me guiaron y dieron acceso a los diferentes Archivos consultados. Especialmente, a Fabiana Bugliani de la Sociedad Argentina de Antropología. Agradezco por último a quienes evaluaron este manuscrito, por sus sugerencias y aportes realizados al mismo.

\section{Referencias Citadas}

Aparicio, F. De 1940. Introducción. Relaciones de la Sociedad Argentina de Antropología 2 (1):1-2.

Arenas, P. 2005. "En la noche de los tiempos". Emilio y Duncan Wagner en el campo de profesionalización de la arqueología. Mundo de Antes 4:159-187.

Arias, A.C. 2017. La participación femenina en los primeros años de la Sociedad Argentina de Antropología (1930-1940). Relaciones de la Sociedad Argentina de Antropología 42 (1):181-188.

Arias, A.C. 2019. Coleccionistas y Estudiosas: las Mujeres en la Producción del Conocimiento Cultural y Antropológico de la Argentina (1920-1940). Tesis Doctoral, Facultad de Ciencias Naturales y Museo, Universidad Nacional de La Plata, ciudad.

Babini, J. 1961. Breve historia de la ciencia argentina. En La Ciencia en la Argentina, Perspectivas Históricas, compilado por M. Asúa, pp. 27-43. Centro Editor de América Latina, Buenos Aires.

Barrancos, D. 2000. Itinerarios científicos femeninos a principios de siglo XX: solas, pero no resignadas. En La Ciencia en la Argentina entre Siglos. Textos, Contextos, Instituciones, compilado por M. Montserrat, pp. 127-144. Manantial, Buenos Aires.

Bonnin, M. 2008. Arqueólogos y aficionados en la Universidad Nacional de Córdoba (Argentina): décadas de 1940 y 1950. Arqueoweb. Revista sobre arqueología en internet. $\mathrm{N}^{\circ} 10: \mathrm{s} / \mathrm{p}$.

Browman, D.L. 2007. La Sociedad Arqueológica de Bolivia y su influencia en el desarrollo de la práctica arqueológica en Bolivia. Nuevos Aportes 4:29-54.

Buchbinder, P. 1997. Historia de la Facultad de Filosofía y Letras. EUDEBA, Buenos Aires.

Buchbinder, P. 2005. Historia de las Universidades Argentinas. Sudamericana, Buenos Aires.

Carrizo, S. 2014. Puntos, líneas y rombos proyectados en el biosólido craneal: los inicios de la trayectoria académica de José
Imbelloni en la antropología argentina. En Antropologías Argentinas. Determinaciones, Creatividad y Disciplinamientos en el Estudio Nativo de la Alteridad, compilado por R. Guber, pp. 43-86. Ediciones al Margen, La Plata.

Centro Argentino de Etnología Americana (CAEA) 1985. Evolución de las Ciencias en la República Argentina 1872. 1972. Tomo X, Antropología. CAEA, Buenos Aires.

Ceruti, C.N. 2012. Avatares de la colección arqueológica del Arroyo Leyes (Depto. Garay, Provincia de santa Fe, Argentina) o la objetividad científica puesta a prueba. Actas el V Congreso Nacional de Arqueología Histórica, Tomo II, pp. 206-235. Editorial Académica Española, Saarbrücken.

Curto, S., G. Jáuregui, M. Escuela, M. Lascano y H. Pena 2008. La fundación de GAEA Sociedad Argentina de Estudios Geográficos - 1922. Boletín de GEA 126:1-49.

Curto, S. y M. Lascano 2014. Elina González Acha de Correa Morales, intelectual y académica. Anales de la Academia Nacional de Geografía 35:27-70.

Di Benedetto, A. 1947. Noticiero argentino. Boletín Bibliográfico de Antropología Americana (1937-1948) 10:9.

Farro, M. 2016. Sociedad científica argentina. En Diccionario Histórico de las Ciencias de la Tierra en la Argentina, coordinado por I. Podgorny y A. Pupio, pp. 368-370. Prohistoria Ediciones, Rosario.

Gänger, S. 2014. Relics of the Past. The Collecting and Study of Pre-Columbian Antiquities in Peru and Chile, 1837-1911. Oxford University Press, Oxford.

García, S.V. 2010. Enseñanza Científica y Cultura Académica. La Universidad de La Plata y las Ciencias Naturales (19001930). Prohistoria Ediciones, Rosario.

García, S.V. 2011. Museos provinciales y redes de intercambio en la Argentina. En Colecionismos, Práticas de Campo e 
Representações, compilado por M.M. Lopes y A. Heizer, pp. 77- 94. Universidade Estadual da Paraíba, Campina Grande.

García, S. 2016. Asociaciones de estudios geográficos y Sociedad Argentina de Ciencias Naturales. En Diccionario Histórico de las Ciencias de la Tierra en la Argentina, coordinado por I. Podgorny y A. Pupio, pp. 57-59 y 368. Prohistoria Ediciones, Rosario.

García, S. e I. Podgorny 2000. El sabio tiene una patria: la Gran Guerra y la comunidad científica argentina. Ciencia Hoy 10 (55):24-34.

Guber, R. 2006. Linajes ocultos en los orígenes de la antropología social de Buenos Aires. Avá. Revista de Antropología 8:1-35.

Guber, R. 2011. Ciro René Lafón y su Pequeña Historia del Museo Etnográfico y la antropología de Buenos Aires. Corpus. Archivos Virtuales de la Alteridad Americana 1 (2):1-23.

Guillemain, H. y N. Richard 2016. Introduction. Towards a Contemporary Historiography of Amateurs in Science (18th-20th Century). Gesnerus 73 (2):201-237.

Halperín Donghi, T. 1962. Historia de la Universidad de Buenos Aires. Eudeba, Buenos Aires.

Hahn, R. 1971. The Anatomy of a Scientific Institution: the Paris Academy of Sciences, 1666-1803. Univ. of California Press, Berkeley.

Kohlstedt, S.G., M.M. Sokal y B.V. Lewenstein 1999. The Establishment of Science in America: 150 Years of the American Association for the Advancement of Science. Rutgers University Press, New Brunswick.

Lascano, M. y S. Curto 2015. El territorio como puente entre la cultura y la política, parte II. El pensamiento de Federico A. Daus 1922-1957. Revista do Departamento de Geografia 28:1-24.

Lopes, M.M. 1999. Aspectos da Institucionalização das Ciências Naturais no Brasil, no Século XIX. Quipu XII (2):217-230.

Mailhe, A. 2016. Polémicas ideológicas en la antropología argentina: el americanismo cientificista de la Biblioteca Humanior. Ponencia presentada en el IX Jornadas de Sociología de la UNLP. Universidad Nacional de La Plata. Facultad de Humanidades y Ciencias de la Educación. Departamento de Sociología, La Plata.

Martínez, A.T., C. Taboada y A. Auat 2011. Los Hermanos Wagner, Entre Mito, Ciencia y Poesía. Universidad Nacional de Quilmes, Bernal.

Mora Nawrath, H. 2016. La Institucionalización de las Ciencias Antropológicas en Chile. Una Aproximación a las Dinámicas Socio-
Organizativas y Cognoscitivas en la Conformación del Espacio Científico (1860 y 1954). Tesis de doctorado. Universidad Nacional de La Plata, Facultad de Humanidades y Ciencias de la Educación, La Plata.

Morínigo, M. 1968. Enrique Palavecino (1900-1966). Cuadernos del Instituto Nacional de Antropología 7:425-428.

Pegoraro, A. 2009. Las Colecciones del Museo Etnográfico de la Universidad de Buenos Aires: un Episodio en la Historia del Americanismo en la Argentina 1890-1927. Tesis de doctorado. Facultad de Filosofía y Letras, Universidad Nacional de Buenos Aires, Buenos Aires.

Podestá, M. 2007. 70 años en la vida de la Sociedad Argentina de Antropología. Relaciones de la Sociedad Argentina de Antropología XXXII:9-32.

Podgorny, I. 2002. Ser todo y no ser nada: paleontología y trabajo de campo en la Patagonia argentina a fines del siglo XIX. En Historia y Estilos de Trabajo de Campo en Argentina, compilado por S. Visacovsky y R. Guber, pp. 31-77. Antropofagia, Buenos Aires.

Podgorny, I. 2004. Tocar para creer. La arqueología en la Argentina, 1910-1940. Anales del Museo de América 12:147-163.

Podgorny, I. 2005. La derrota del genio. Cráneos y cerebros en la filogenia argentina. Saber y Tiempo 5 (20):63-106.

Podgorny, I. 2014. Sobre la constitución de los objetos etnológicos en los inicios del siglo XX: museos, falsificaciones y ciencia. Museologia e Interdisciplinaridade III (5):21-35.

Pupio, A. 2013. Archivos para una historia de la práctica de la arqueología. Revista Electrónica de Fuentes y Archivos 4:24-33.

Pyenson, L. y S. Sheets Pyenson 1999. Servants of Nature: A History of Scientific Institutions, enterprises, and sensibilities. Norton and Company, New York-London.

Soprano, G. 2006 Configuración de liderazgos y grupos académicos en la investigación antropológica argentina. Análisis histórico centrado en la Facultad de Ciencias Naturales y Museo de la Universidad Nacional de La Plata. 1930-1990. Ponencia presentada en el Primer Congreso Argentino de estudios Sociales de la Ciencia y la Tecnología, s/p. Universidad Nacional de Quilmes, Bernal.

Soprano, G. 2009. La Antropología Física entre la universidad y el Estado. Análisis de un grupo académico universitario y sus relaciones con las políticas públicas del Instituto Étnico Nacional (1946-1955). Estudios Sociales 37:63-95.

\section{Notas}

${ }^{1}$ En el sentido también que usan Pyenson y Sheets-Pyenson (1999) para analizar las sociedades científicas surgidas en el siglo XVII. Ver también Kohlstedt et al. 1999, entre otros.

${ }^{2}$ La Sociedad no cuenta con un archivo propiamente dicho, pero en el último tiempo se han tratado de reunir y conservar los documentos que han sobrevivido del funcionamiento de esta asociación. Estos materiales incluyen los libros de actas desde su fundación hasta el presente, correspondencia, registros de socios, registros contables y movimientos de caja, folletos de actividades, certificados de envíos e intercambio de la revista Relaciones de la Sociedad Argentina de Antropología, separatas y originales de dicha revista, fotografías, memorias, estatutos, entre otros. En este artículo los documentos consultados aquí se refieren como Archivo de la Sociedad Argentina de Antropología (ASAA).

${ }^{3}$ Luego Academia Nacional de Historia.
${ }^{4}$ Sobre su trayectoria, ver, entre otros: Barrancos (2000), Zusman (2001), Curto y Lascano (2014), García (2016).

5 "Nuestro Programa" en Revista de la Sociedad de Amigos de la Arqueología, Tomo I, 1927, pp. 5-6, Montevideo.

${ }^{6}$ Boletín Bibliográfico de Antropología Americana (19371948), Vol. 1, No. 4 (octubre a diciembre, 1937), pp. 199-202.

7 Boletín Bibliográfico de Antropología Americana (19371948), Vol. 5, No. 1/3 (enero a diciembre, 1941), pp. 9.

${ }^{8}$ Copiador de la SAA 1936-1943, AFDME.

${ }^{9}$ Acta de fundación de la Sociedad, 24 de abril de 1936 (Libro de Actas, 1936, s/n, f. 4). Archivo SAA.

${ }^{10}$ Francisco de Aparicio (1892-1951) fue profesor de Historia y Geografía Americana en la Facultad de Ciencias de la Educación de la Universidad del Litoral en Paraná (19201930) y entre fines de 1930 y 1947 profesor de Arqueología Americana en Filosofía y Letras en Buenos Aires; profesor de 
Historia en el Colegio Nacional de Buenos Aires entre 1933 y 1946, y director del Museo Etnográfico entre 1937 y 1947 (Guber 2006).

${ }^{11}$ Eduardo Casanova (1902-1977), oriundo de España, cursó la carrera de profesorado secundario con especialidad historia en la Facultad de Filosofía y Letras de la Universidad de Buenos Aires, egresando en 1929. Ejerció la docencia secundaria y fue profesor de Arqueología Americana de la Facultad de Filosofía y Letras entre 1939 y 1948 como adjunto y desde 1949 como titular. También fue jefe de la sección arqueología del Museo Argentino de Ciencias Naturales "Bernardino Rivadavia" entre 1930 y 1946. Ver más en: "Casanova, Eduardo", en Quién es en quién en la República Argentina. Biografías contemporáneas, Editorial Guillermo Kraft Ltda., Buenos Aires, 1995, pp. 147.

${ }^{12}$ José Imbelloni (1885-1967). Italiano, estudió Medicina en la Universidad de Perugia. En 1921 ocupó el puesto de profesor suplente de Antropología en la Facultad de Filosofía y Letras (UBA), de la cual fue Profesor titular en 1939. Desde 1922 fue encargado de las investigaciones antropológicas en el Museo Etnográfico. En 1946 fue designado director del Museo Etnográfico. En 1947 fue nombrado Director del Instituto de Antropología, también dependiente de la Facultad de Filosofía y Letras. Ver, entre otros: CAEA (1985), Carrizo (2014), Mailhe (2016).

${ }^{13}$ Fernando Márquez Miranda (1897-1961), estudió abogacía (egreso 1918) y profesorado de historia (egreso 1923) en la Facultad de Derecho y Ciencias Sociales de La Plata y obtuvo un diploma de doctor en historia en España (Madrid, egreso 1936). Desempeñó cargos docentes en diferentes instituciones, como la Facultad de Filosofía y Letras de Buenos Aires (Prehistoria y Arqueología Americana, entre 1939-1947 y entre 1955-1961), en la Facultad de Humanidades y Ciencias de la Educación (Prehistoria y Arqueología y Americana, 1923, 1927, 1930, 1932-1947 y 1955-1961) y en el Museo de La Plata (Arqueología y Etnografía, 1942-1947 y 1955-1961) donde fue Jefe de la Sección de Etnografía y Arqueología entre 1933 y 1947, quedando cesante a inicios de 1947. Tras la Revolución Libertadora volvería a trabajar en el Museo, llegando a dirigir esta institución entre 1955 y 1957.

${ }^{14}$ Enrique Palavecino (1900-1966) no contaba con formación universitaria ni había concluido los estudios secundarios, pero comenzó sus actividades científicas, al igual que otros jóvenes, asistiendo como adscripto a las secciones del Museo de Ciencias Naturales de Buenos Aires. Allí realizó trabajos anatómicoantropológicos para luego orientarse hacia temas lingüísticos, etnográficos y folklóricos (Morínigo 1968, Soprano 2006).

${ }^{15}$ Félix Outes (1878-1939), realizó estudios en la Facultad de Derecho y Ciencias Sociales y en la Facultad de Medicina de la Universidad de Buenos Aires, sin completar ninguna carrera. Fue profesor adjunto de Etnografía en la Facultad de Ciencias Naturales de La Plata (1906-1911); profesor suplente de Antropología en la misma facultad; profesor Adjunto de Arqueología; profesor de Antropología en la Facultad de Filosofía y Letras de Buenos Aires; profesor interino de Geografía Humana en esa facultad -luego titular de la materia-; director del Instituto de Investigaciones Geográficas; director y jefe de expediciones arqueológicas del Museo Antropológico y Etnográfico (1930-1938); profesor suplente de Antropología en la Facultad de Ciencias Exactas, Físicas y Naturales de la Universidad Nacional de Buenos Aires y profesor en el Colegio Nacional de Buenos Aires. Sucedió a Debenedetti (1917-1930) en la dirección del Museo Etnográfico de Buenos Aires (CAEA 1985, Guber 2006).

${ }^{16}$ Milcíades Alejo Vignati (1895-1978), se recibió como maestro normal en 1915 y de Profesor normal de Ciencias en 1918. Cursó algunas materias en la Facultad de Ciencias Exactas, Físicas y Naturales de Buenos Aires. Fue docente de Antropología en la Facultad de Ciencias Exactas, Físicas y Naturales de Buenos
Aires de 1930 a 1938, adscrito al Museo Etnográfico de Buenos Aires en 1926, 1927 y de 1930 a 1932; y luego profesor y jefe del Departamento de Antropología del Museo de La Plata entre 1930 y 1955. Se ocupó de temas relativos a antropología física, arqueología, prehistoria, etnología y lingüística (Pupio 2013, AHMLP).

${ }^{17}$ En la primera sesión, el 4 de mayo de 1936, se invitó a incorporarse como socios activos y como parte de la Comisión Directiva a Serrano, Emilio y Duncan Wagner y a Héctor Greslebin. Este último fue el único que no aceptó la invitación, por "razones de índole particular". Memoria de la SAA 193637, en Relaciones Tomo I, 1937, pp. 201.

${ }^{18}$ Libro de Actas, 1936, pp: 4, ASAA

${ }^{19}$ Crónica oficial, en Relaciones, Tomo I, 1937, pp. 198.

${ }^{20}$ Mientras en 1936 se había establecido una cuota de treinta pesos anuales, en 1937 pasó a ser de diez pesos anuales.

${ }^{21}$ Memoria de la Sociedad Argentina de Antropología, 1937-38. En Relaciones, Tomo I, 1937, pp. 203.

${ }^{22}$ En esta asociación, como en otras sociedades científicas argentinas, las mujeres fueron admitidas sin que se establecieran criterios diferentes en las membresías para socios masculinos o femeninos. Véase al respecto la figura 4 , en el cual se muestra la proporción de socios y socias. El aumento progresivo de la membresía femenina ha sido analizado en Arias (2017).

${ }^{23}$ En un listado de 1940 , de un total de 181 miembros 81 son de Capital, 5 de La Plata, 3 de Olivos; 10 repartidos de a 2 en Ramos Mejía, Rosario, Santa Fe, Santiago del Estero y Temperley y 18 restantes en diferentes localidades del país: Barranqueras (Chaco), Bernal, Concepción (San Juan), Godoy Cruz, Mendoza, Merlo, Munro, Paraná, Pergamino, Salta, San Andrés, San Fernando, San Isidro, Santa Rosa, Tigre, Tucumán, Vicente López y Wilde.

${ }^{24}$ Este año ingresan 49 nuevos miembros, siendo 36 activos (31 varones y 5 mujeres) y 13 estudiantes ( 8 varones y 5 mujeres). Cabe recordar que en las primeras décadas del siglo XX la mayoría de los alumnos de los cursos de antropología y arqueología, tanto en la Universidad de Buenos Aires (Buchbinder 1997) como en La Plata (a partir de 1920) pertenecían a las carreras de profesorado, con un alto porcentaje de mujeres (García 2010).

${ }^{25}$ Este año ingresaron 67 asociados, de los cuales 21 eran socios activos (15 varones y 6 mujeres) y 46 socios estudiantes (19 varones y 27 mujeres).

${ }^{26}$ Por ejemplo, el 22 de junio de 1939 se hace constar en actas la renuncia (sin motivos explicitados en el acta) de los siguientes socios activos: Alfredo Taillard, Oscar Vigliani, Osvaldo Loudef, José Anesi, Martín Noel, Rómulo Zabala y Emilio Wagner (Archivo SAA).

${ }^{27} \mathrm{La}$ ausencia de registros de asociados por año dificulta la elaboración de cálculos más precisos respecto de los miembros que se dieron de baja.

${ }^{28}$ Boletín de la SAA, 1943: 56.

${ }^{29}$ Año a año, los socios estudiantes aumentan en general, y también aumenta la proporción femenina: en 1938 ingresan 4 personas (3 mujeres y 1 varón), 16 en 1939 (13 mujeres y 3 varones), 26 en 1940 ( 17 mujeres y 9 varones), 46 en 1941 ( 27 mujeres y 19 varones) y 20 en 1942 (12 mujeres y 8 varones). En la figura 4 se presentan las proporciones totales de varones y mujeres en el período 1936-1943.

${ }^{30}$ Crónica Oficial de la SAA, en Relaciones $\mathrm{N}^{\circ} 2,1942$.

${ }^{31}$ En 1937 se realizaron cuatro sesiones de comunicaciones (julio, agosto, septiembre y noviembre), en 1938 se realizaron 5 sesiones (mayo, junio, julio, septiembre y octubre), en 1939 se realizaron 6 sesiones (abril, mayo, agosto, octubre, noviembre y diciembre), en 1940 se realizaron 7 sesiones (abril, mayo, junio, julio, agosto, septiembre y octubre) y 4 sesiones en 1941 (abril, junio, agosto y septiembre).

${ }^{32}$ Memoria de la Sociedad Argentina de Antropología, 193738. En Relaciones, Tomo I, 1937, pp. 200. 
${ }^{33}$ Cabe señalar que la Sociedad Argentina de Estudios Geográficos realizaba desde 1931 la "Semana de la Geografía".

${ }^{34}$ A diferencia de otras "semanas" científicas, como la Semana de la Geografía o la Semana del Mar, en la antropológica no se realizaban exposiciones visuales, paneles ni muestrarios de objetos y colecciones.

${ }^{35}$ Crónica Oficial de la SAA, en Relaciones $N^{\circ} 2,1942$.

${ }^{36}$ Archivo SAA.

${ }^{37}$ Luego, en 1957 y en 1960, la Sociedad organizó las llamadas

"Jornadas Internacionales de Antropología y Etnografía".

${ }^{38}$ Luego será retomada en 1970, publicándose hasta el presente con números por lo general anuales.

${ }^{39} 1 / 9 / 1936$ Copiador SAA 1936-1943, AFDME.

${ }^{40}$ En 1944, el subsidio era de 2000 pesos anuales y no alcanzaba a pagar los costos totales del Boletín más la revista Relaciones. Ello generaba deudas con las imprentas y reiteradas solicitudes a los autores de trabajos para que respeten el número máximo de páginas. En 1944 la sociedad tenía una deuda con la Imprenta Plantié que sumaba 6.349 pesos. ASAA, Libro de Actas $\mathrm{N}^{\circ} 1$, p. $98,99,101$

${ }^{41}$ Por ejemplo, el Boletín publicado en 1944 fue costeado gracias a una publicidad de la Editorial Emecé. ASAA, 25/8/1944, Libro de Actas $\mathrm{N}^{\circ} 1$, p. 99.

${ }^{42}$ Memoria de la SAA, 1937-38, en Relaciones, tomo II, 1940, pp. 257.

${ }^{43}$ Este último fue solicitado para su dictado al Foto Club Argentino.

${ }^{44}$ Carta a Jorge E. Coll, Ministro de Justicia e Instrucción Pública, 24/8/1938. Copiador SAA 1936-1943, AFDME.

${ }^{45}$ Libro de Actas, 1939, pp: 51, Archivo SAA.

${ }^{46}$ Libro de Actas, 1939, pp. 53, Archivo SAA.

${ }^{47}$ Ambas propuestas fueron realizadas en mayo de 1938.

${ }^{48}$ Relaciones, tomo II (1940:248), discurso de Casanova en homenaje a Ambrosetti y Outes.

${ }^{49}$ El libro propone que la provincia de Santiago del Estero había sido poblada, en una lejana época, por pueblos de una "civilización adelantada", servidores de una deidad constituida por la trinidad hombre-ave-serpiente. Las cerámicas eran correlacionadas por los Wagner con otras de Eurasia y América, tanto en sus formas peculiares como por su simbolismo, evidenciando para ellos el paralelismo entre Troya y Santiago del Estero (Arenas 2005).

${ }^{50}$ La mayoría de las veces fue Zapata Gollán quien rechazó las excavaciones, debido a la creciente del río Paraná (1942), "por falta de medios de locomoción, aún de propiedad particular, que estuvieran en condiciones de hacer los viajes diarios entre Santa Fe y el yacimiento de Leyes" y porque los terrenos han sido sembrados recientemente y eso impediría los trabajos (1945) y por haber sido desalojado el local que ocupaba el Departamento de Estudios Etnográficos y Coloniales (1947). Copiador de la SAA, AFDME. Los fondos destinados a las investigaciones en el Arroyo Leyes fueron utilizados en las actividades de la octava Semana de la Antropología, que se realizó en 1951 en dos sesiones, una en Buenos Aires y otra en Santa Fe.

${ }^{51}$ Boletín de la SAA, agosto de 1943: 61-62. E1 9 de junio de 1943 se realizó una reunión de comunicaciones donde Aparicio presentó un plan de investigación intensiva de los
Valles Calchaquíes, el resumen del mismo fue publicado en el citado Boletín de agosto de 1943. Además, la noticia señalaba que se pretendía realizar un "trabajo científico, sistemático y colectivo, en el cual trabajarían a la par alumnos y profesores." El verano anterior se habían realizado expediciones de modo preliminar, por parte de personal del Museo; junto a Aparicio, viajaron los profesores Augusto Raúl Cortazar, Alberto M. Salas, Lía Raquel Sanz de Arechaga y el estudiante Juan Antonio Güemes. En este viaje hallaron una "ciudad indígena intacta", en Tolombón, Valle de Santa María, Salta.

${ }^{52}$ Libro de Actas $\mathrm{N}^{\circ}$ 1, 1945, pp:106-107, ASAA.

${ }^{53}$ Libro de Actas $\mathrm{N}^{\circ} 1,1945$, pp:106-107, ASAA.

${ }^{54}$ María de las Mercedes Constanzó, nació en Santa Fe en 1909 y se graduó como Profesora de Historia en la Facultad de Filosofía y Letras de Buenos Aires en 1932, doctorándose ocho años después en la misma facultad. Se dedicó a la docencia secundaria y se desempeñó como técnica del Museo Etnográfico desde 1940. En 1946 fue convocada para "reestructurar" el Instituto de Antropología de la Universidad de Tucumán (en reemplazo de Enrique Palavecino), asumiendo interinamente la dirección hasta 1947. También actuó como profesora de Prehistoria y Etnografía en la Facultad de Filosofía y Letras de Tucumán entre 1946-1947. Sus investigaciones se dedicaron a la antropología física, en línea con aquellos temas desarrollados por Imbelloni, del cual fue asistente. Principalmente se dedicó al estudio de los restos óseos de los indígenas prehispánicos, describiendo y estudiando las lesiones dentarias y anomalías que permiten establecer las enfermedades que padecieron (Arias 2019).

${ }_{55}^{55}$ Libro de Actas, $\mathrm{N}^{\circ} 1$, p. 114, 10 de abril de 1947, ASAA.

56 "Noticiario argentino". Por Antonio Di Benedetto. En Boletín Bibliográfico de Antropología Americana (1937-1948), Vol. 10 (enero-diciembre, 1947), pp. 9-15.

${ }^{57}$ Libro de Actas $\mathrm{N}^{\circ} 1$, p. 119,12 de abril de 1948, ASAA.

${ }^{58}$ Libro de Actas $\mathrm{N}^{\circ} 1$, p. 122, 123, 12 de abril de 1948, ASAA.

${ }_{59}$ Junta Directa de la SAA, "Reiniciando el camino", Boletín $\mathrm{N}^{\circ} 10$, agosto de 1953.

${ }^{60}$ En este círculo los propósitos consistían en: “a) Fomentar la amistad y colaboración entre los estudiantes del Doctorado de Historia y cuantos aspiren a realizar obra original en el campo de las "Ciencias del Hombre". b) Mantener el contacto con los profesores transcurrido el periodo escolar, esta vez como consejeros y amigos. c) Estimular la realización de trabajos de investigación. d) Proporcionar la oportunidad de exponer trabajos monográficos, obteniendo con ello, a la par de una justa satisfacción personal, la fluidez y soltura necesarias para la enseñanza. e) Realizar sesiones de comentario bibliográfico para mantener a los asociados al tanto de los trabajos recientemente aparecidos. f) Organizar viajes de estudio." Ver "Constituyóse el Centro AKIDA, Estudiantes de Historia", en AMICITIA, Año III, No 16, Agosto de 1943, pp. 20.

${ }^{61} \mathrm{El}$ nombre viene de AKIS -en griego: estímulo y punta de proyectil o acicate-, pero también de las iniciales de los profesores de segundo año de la carrera de Historia en Buenos Aires: Ardissone, Constanzó, Imbelloni, Daus, Aparicio (Guber 2011).

62 "Noticiario argentino", Di Benedetto (1947). 
